\title{
¿Son las investigadoras de Documentación más interdisciplinarias frente a sus colegas hombres? Un análisis aplicado al profesorado español
}

\author{
Manuel Blázquez Ochando* \\ Michela Montesi* \\ Isabel Villaseñor Rodríguez*
}

Artículo recibido:

29 de marzo de 2021

Artículo aceptado:

28 de junio de 2021

Artículo de investigación

\section{Resumen}

La actividad científica desempeñada por mujeres difiere bajo varios puntos de vista de la de los hombres, tanto en términos de producción como de impacto. En este trabajo, comparamos la actividad científica de 349 profesores y profesoras del área de Biblioteconomía y Documentación (ByD) afiliados con universidades españolas, con base en el análisis de su trayectoria de publicación en revistas vaciadas en Scopus. Se analizan las publicaciones de la muestra atendiendo a tres indicadores de interdisciplinariedad (variedad, equilibrio y disparidad), y midiendo la disparidad a través de métodos de Procesamiento del Lenguaje Natural

* Facultad de Ciencias de la Documentación, Universidad Complutense de Madrid, España manublaz@ucm.es mmontesi@ucm.es isavilla@ucm.es

INVESTIGACIÓN BIBLIOTECOLÓGICA, vol. 35, núm. 89, octubre/diciembre, 2021, México, ISSN: 2448-8321 pp. 133-149 
(PNL) y Recuperación de Información (RI). A pesar de no encontrar diferencias importantes en las tres dimensiones, los datos obtenidos apuntan a la posible inadecuación de los índices de citas de reflejar las actividades académica desempeñadas por las mujeres en toda su variedad, así como a la necesidad de comparar la producción de hombres y mujeres con base en el análisis temático de los contenidos producidos.

Palabras clave: Interdisciplinariedad; Producción Científica; Sesgo de Género; Análisis Temático; Biblioteconomía y Documentación

Are Information Science female researchers more interdisciplinary than their male colleagues? An analysis of Spanish faculty

Manuel Blázquez Ochando, Michela Montesi and Isabel Villaseñor Rodríguez

\section{Abstract}

The scientific activity of women differs in several ways from men's, and several studies have found differences in terms of production and impact. The present work compares the research activity of 349 LIS professors with a Spanish affiliation by studying their Scopus publications. The sample is analyzed from an interdisciplinary perspective in terms of variety, balance and disparity. Disparity was measured through methods of NLP and IR. Despite not finding important differences in the three dimensions for male and female professors, the data obtained point to the possible inadequacy of citation indexes with respect to the spectrum of academic activities carried out by women in all its variety, as well as the need to compare the production of men and women based on the thematic analysis of the contents produced.

Keywords: Interdisciplinarity; Scientific Production; Gender Bias; Thematic Analysis; Library and Information Science 


\section{INTRODUCCIÓN}

A unque de forma no consistente, la literatura especializada ha documentado diferencias en la labor científica desempeñada por las mujeres respecto a la de los hombres, tanto en términos de producción, especialización y colaboración, por el lado de la actividad, como en términos de citación y visibilidad, por el lado del impacto. Según la investigación previa, las mujeres investigadoras serían menos productivas (Larivière et al., 2011; Larivière et al., 2013; European Commission, 2018; Hernández-Martín et al., 2019) y menos especializadas que los hombres (Leahey, 2006), mientras que sus patrones de colaboración serían más abiertos, cooperativos entre géneros e interdisciplinarios (Rhoten y Pfirman, 2007). Por el lado del impacto, la producción femenina, liderada por mujeres o realizada con una proporción mayor de mujeres tiende a citarse menos (Knobloch-Westerwick y Glynn, 2013; Chan y Torgler, 2020), incluso cuando se publica en revistas similares en términos de impacto (Beaudry y Larivière, 2016). Aunque los resultados de diferentes estudios no son coherentes en este sentido y algunos no detectan diferencias de impacto entre hombres y mujeres (Thelwall y Nevill, 2019), en general el trabajo de investigación femenino parece citarse con menor intensidad, por lo menos en muchas áreas. El sesgo de género se produce también en la comunicación científica en los medios sociales, donde la presencia de las mujeres sería en general menos visible que la de los hombres (Sugimoto et al., 2017; Montesi, Rodríguez Villaseñor y Bittencourt Dos Santos, 2019). Las diferencias en términos de visibilidad e impacto, así como las documentadas en términos de producción y actividad, tienen repercusiones obvias en los procesos de evaluación, donde se enfatizan precisamente todas estas dimensiones. En el presente trabajo pretendemos ahondar en las peculiaridades de la actividad científica de las mujeres analizando las dinámicas de publicación de profesoras y profesores de Biblioteconomía y Documentación (en adelante ByD) afiliados con universidades españolas. Concretamente, nos centramos en los procesos de difusión del conocimiento y planteamos la cuestión de si las mujeres publican investigaciones más interdisciplinarias que los hombres. El trabajo de Jamali, Abbasi y Bornmann (2020) confirma la relación entre género e investigación interdisciplinaria (IID) en un conjunto de publicaciones del profesorado australiano en las áreas de Física, Química y Biología en el periodo 1980-2014, obtenidas de WoS. Sin embargo, la relación entre género e interdisciplinariedad no cuenta todavía con una base de evidencia sustancial.

Los paralelismos entre la IID y la investigación realizada por mujeres llevan a Rhoten y Pfirman (2007) a plantearse la cuestión de si existe un cruce 
entre estos dos ámbitos. Según estas autoras, las mujeres se sienten atraídas naturalmente hacia la IID por una mayor inclinación a buscar conexiones entre ideas y contextos, sus menores vínculos institucionales con las normas del sistema de producción de conocimiento articulado en disciplinas, y por una epistemología basada en la racionalidad "afectiva", la interconexión y el holismo. Tanto la IID como la investigación realizada por mujeres son a menudo objeto de discriminación en los procesos de revisión y evaluación. A través del caso de más de 18000 solicitudes enviadas al Australian Research Council's Discovery Programme, Bromham, Dinnage y Hua (2016) demuestran esta desventaja para la IID pues encontraban que, a mayor grado de interdisciplinariedad de las propuestas, correspondía una menor probabilidad de financiación. Tanto la investigación llevada a cabo por mujeres como la IID pueden tener salida en revistas de menor impacto o recibir menos citas en casos como, por ejemplo, la investigación sobre las aguas subterráneas (Barthel y Seidl, 2017). Nielsen (2017) documenta diferencias a favor de los hombres en los procesos de evaluación de la comunidad científica danesa, que achaca a la escasa participación de las mujeres en los comités evaluadores, por un lado, y a la ventaja que el sistema asigna a la investigación colaborativa.

Por otro lado, existe un énfasis importante en promover desde la política tanto la IID como la investigación de mujeres (Rhoten y Pfirman, 2007). Se supone que ambos tipos de investigación pueden contribuir a la resolución de problemas del mundo real y así lo entienden muchas agencias financiadoras (Jacobs y Frickel, 2009). Repasando cuestiones pendientes con respecto a la IID, Jacobs y Frickel (2009: 52) constataban que la "interdisciplinarity is gendered" y que igual de importantes que las motivaciones sociales que llevan a promocionarla son las consecuencias de ésta, haciendo referencia en particular a los women studies y a otros dominios de IID marginalizados. En este sentido, cabe añadir que tanto la IID como la investigación de mujeres presentan niveles de productividad inferiores respecto al resto de investigaciones (Leahey, Beckman y Stanko, 2017) y, aunque el mayor calado social de estos dos tipos de investigación no se suele mencionar para explicar los niveles de producción inferiores, tampoco existen evidencias de lo contrario.

Finalmente, los propios patrones de colaboración de las mujeres podrían apuntar a una mayor inclinación hacia la diversidad respecto a los hombres, pues, en algunos estudios, tienden a ser más inclusivos en términos de género (Ghiasi, Harsh y Schiffauerova, 2018; Araújo et al., 2017; Whittington, 2018), pudiendo resultar en una actitud más inclusiva también en términos disciplinarios. Asimismo, la colaboración de las mujeres sería más local, debido, en parte, a las necesidades de la conciliación familiar (Larivière et al., 2011; Abramo, D’Angelo y Murgia, 2013). Cabe preguntarse si también son igual de 
locales y cercanos los problemas de investigación que se plantean las mujeres investigadoras, así como las repercusiones de su investigación en la realidad más próxima; la investigación de Abramo, D’Angelo y Di Costa (2019) apoya, aunque moderadamente, la relación entre localidad de las redes de colaboración y la inclinación hacia una investigación más diversificada.

En el marco de estos paralelismos nos planteamos la cuestión de si las mujeres investigadoras son más interdisciplinarias que los hombres. En el ámbito de la Lingüística y la Sociología, Leahey (2006) encontraba que la mayor productividad de los hombres podía achacarse a su mayor especialización frente a la mayor diversificación de las mujeres. Por otro lado, Abramo, D’Angelo y Di Costa (2018), tras analizar la producción de investigadores italianos de Ciencias en el periodo 2004-2008, concluían que las diferencias entre hombres y mujeres en términos de producción no podían explicarse por una mayor o menor inclinación hacia la diversificación de las temáticas estudiadas. En Woolley et al. (2014) tampoco el género influye en la inclinación hacia la IID; sin embargo, otros estudios han comprobado la existencia de una conexión entre género e interdisciplinariedad (Van Rijnsoever y Hessels, 2011; Araújo et al., 2017; Meng y Shapira, 2010) y recientemente Jamali, Abbasi y Bornmann (2020) concluían que las mujeres de universidades australianas presentaba una mayor inclinación a publicar investigación diversificada temáticamente en las áreas de Biología, Física y Química.

\section{Medición de la interdisciplinariedad}

Existen diferentes formas de analizar y medir la interdisciplinariedad de autores, publicaciones, revistas u otras entidades dentro del sistema de comunicación científica. Para determinar la pertenencia a cierto ámbito disciplinario, se suelen emplear los sistemas de clasificación en categorías de WoS y Scopus (que contemplan respectivamente cerca de 250 y 340 categorías respectivamente), u otros sistemas de clasificación como el ECOOM Leuven-Budapest Subject Classification que Zhang, Rousseau y Glänzel (2016) y Zhang et al. (2018) utilizan por incluir un número ni demasiado grande ni demasiado pequeño de grandes áreas (16) y sub-áreas (68), o el sistema de clasificación del profesorado italiano en 370 sectores científicos y 14 grandes áreas disciplinarias que aprovechan Abramo, D’Angelo y Zhang (2018). Solomon, Carley y Porter (2016) explican que las métricas de interdisciplinariedad pueden medir tanto la "integración" de conocimiento en el ámbito de las referencias citadas en artículos individuales -y, por extensión, en revistas, áreas de investigación o instituciones- como la "difusión" de conocimiento, en términos de citación de los artículos de un conjunto documental concreto 
por parte de revistas pertenecientes a categorías diferentes. En ambos casos, la medición de la interdisciplinariedad suele atender a tres dimensiones diferentes de la integración y difusión del conocimiento: la variedad o el número de diferentes categorías en las cuales se asignan los elementos del sistema (referencias, citas o publicaciones, por ejemplo); el equilibrio o la uniformidad en la distribución de los elementos del sistema en las diferentes categorías, y la distancia o la disparidad entre los elementos analizados, se observa mayor disparidad entre las referencias, citas o publicaciones que se producen en categorías muy poco afines y distantes la una de la otra. Las tres dimensiones se pueden integrar en un único indicador, como el indicador Rao-Stirling Diversity (Porter y Rafols, 2009) o el Integrated Diversity Index, desarrollado en el trabajo de Zhang, Rousseau y Glänzel (2016) y de Zhang et al. (2018), o se pueden analizar por separado para determinar, por ejemplo, el efecto de cada una de ellas en los patrones de citación de los conjuntos documentales analizados (Wang, Thijs y Glänzel, 2015). Una vez calculados los diferentes indicadores, integrados o no, se pueden recabar estadísticas descriptivas para los conjuntos documentales analizados y ordenar los elementos examinados.

Debido a las varias posibles interpretaciones de la diversidad, los diferentes indicadores pueden devolver resultados poco coherentes (Huang et al., 2021). Zhang et al. (2018) afirman que no existe de momento una metodología definitiva para medir la interdisciplinariedad, al igual que Adams, Loach y Szomszor (2016) y Leydesdorff y Rafols (2011). Como consecuencia de estas limitaciones y de los resultados incoherentes encontrados en estudios previos, Zhang et al. (2018) recomiendan revertir los enfoques de análisis interdisciplinario y, en lugar de contar con clasificaciones predefinidas, sugieren realizar análisis de abajo-arriba, incluyendo, entre varias posibilidades de análisis, la interpretación de los contenidos. En este sentido se orientan los estudios de Gomes y Dewes (2017) y Dong et al. (2018).

\section{Objetivo}

El objetivo del presente trabajo es comparar desde un punto de vista bibliométrico la actividad científica de 349 profesores y profesoras del área de ByD atendiendo a sus patrones de publicación. Si las políticas científicas de género miran a igualar hombres y mujeres, las críticas que reciben los criterios de evaluación normalmente adoptados (productividad e indicadores basados en citación como el factor de impacto) animan a buscar alternativas de evaluación que permitan al colectivo femenino poner en valor ciertas características de su actividad investigadora. Nuestra hipótesis de partida es que pueden existir diferencias en términos de interdisciplinariedad entre las 
publicaciones de las profesoras y las correspondientes a profesores, y pretendemos comprobarlo a través del análisis de su producción científica recopilada en Scopus. Esta base de datos se escogió debido a su mejor cobertura de la producción en idiomas diferentes al inglés.

\section{Metodología}

En el presente estudio, trabajamos con la muestra de 183 profesoras y 166 profesores afiliados con universidades españolas y analizada en un trabajo previo (Montesi, Rodríguez Villaseñor y Bittencourt Dos Santos, 2019). Los 349 individuos de la muestra se buscaron automática y manualmente en la base de datos Scopus para localizar las publicaciones que les correspondían desde 1980 hasta febrero de 2019. Se tuvieron en cuenta todos los tipos de documentos reflejados, en concreto libros y capítulos de libros, artículos en revistas científicas y revistas profesionales, y actas de congresos. Para determinar los indicadores de variedad y equilibrio, se utilizaron las 334 categorías que contempla Scopus, mientras que para la disparidad se atendió a las mismas categorías y además al título y resumen de cada documento. Cuando las revistas están clasificadas en más de una categoría se tuvieron en cuenta todas las categorías en las que aparecía. A partir de esta clasificación y de la descripción de contenido de los documentos, se obtuvieron tres indicadores para todos los individuos de la muestra: variedad, equilibrio y disparidad. La variedad mide la proporción de categorías no vacías para cada individuo en los dos conjuntos, es decir, la proporción de categorías en las cuales cada autor o autora tiene trabajos publicados sobre el total de categorías. En segundo lugar, el coeficiente de Gini se utilizó para medir el nivel de concentración de los trabajos de cada autor en las diferentes categorías temáticas. Normalmente, valores más altos del coeficiente de Gini denotan mayor desequilibrio y concentración de publicaciones en unas pocas categorías temáticas. Por eso, en los resultados, mostramos 1-Gini para que el indicador tenga la misma dirección que los demás y los valores más próximos al 1 denotan mayor interdisciplinariedad. Finalmente, la disparidad de las publicaciones de cada autor se midió atendiendo al contenido de cada artículo. Esto se realizó evaluando el título y el resumen de cada publicación en relación con el resto de artículos de la categoría temática, mediante técnicas de PNL y RI, con base en las cuales se determinó un coeficiente de disparidad de la producción científica de cada individuo (ecuación 1). 


$$
D=1-\frac{\log _{10}\left(\sum_{i j} \operatorname{Sim} \operatorname{Cos}\left(d_{i}, d_{j}\right)\right)}{N}
$$

Donde:

Sim $\operatorname{Cos}=$ Similaridad del coseno

$i=$ Categorías temáticas del autor o autora del que se estudia la disparidad en particular, sin repetición

$j$ = Conjunto de categorías temáticas en las que ha publicado artículos cada individuo de la muestra, sin repetición

$d i=n$ documentos de la categoría $i$

$d j=n$ documentos de la categoría $j$

$N$ = Número total de categorías temáticas en las que han sido clasificados los documentos de todos los autores y autoras de las dos muestras, sin repetición

La disparidad temática de un autor es igual a 1 menos la fracción del logaritmo en base 10 de la suma de los coeficientes de similaridad entre los documentos (calculado con base en su título y resumen) publicados por el autor en una categoría temática dada con respecto al resto de documentos de dicha categoría, publicados por el resto de autores de la muestra a estudiar, dividido por el número total de categorías en las que se han clasificado los documentos de la muestra, conformada por todos los autores estudiados. El resultado que se obtiene es la similaridad absoluta de la producción científica de cada individuo con respecto al resto de la muestra. Al restar 1 a ese coeficiente de similaridad se obtiene la diferencia, es decir, la disparidad.

Los coeficientes obtenidos por cada autor y autora se sometieron a las pruebas de significación pertinentes (prueba U de Mann-Whitney) con el objetivo de rechazar la hipótesis nula de no diferencia entre los dos conjuntos, a través de la herramienta IBM SPSS Statistics. Se escogieron pruebas estadísticas no paramétricas atendiendo a la distribución no normal del conjunto de datos manejados, que no cumplió con los requisitos de normalidad definidos en Stjernholm Madsen (2016: 55-60).

\section{Resultados}

Según puede apreciarse en la Tabla 1, $55 \%$ de las profesoras y $65 \%$ de los profesores tienen por lo menos una publicación en Scopus. Aparte de la diferencia en términos de presencia, es posible apreciar otra en términos de producción, siendo la mediana de publicaciones por individuo inferior en el 
caso de las mujeres (5 frente a 8.5). La diferencia entre los dos grupos en términos de productividad se acerca mucho a niveles de significación (U de Mann-Whitney $=4603$, valor de $p=.051$ ).

\begin{tabular}{|r|r|r|}
\hline & M & \multicolumn{1}{|c|}{ H } \\
\hline Número total de individuos en la muestra & 183 & 166 \\
\hline Profesorado con presencia en Scopus & 101 & 108 \\
\hline \% Profesorado con presencia en Scopus & $55 \%$ & $65 \%$ \\
\hline Número total de publicaciones & 666 & 960 \\
\hline Mediana de publicaciones por persona & 5 & 8.5 \\
\hline Promedio de publicaciones por persona & 8.48 & 14.28 \\
\hline
\end{tabular}

Tabla 1. Datos globales relativos a la muestra estudiada

Según puede apreciarse en los diagramas de las Figuras 1a y 1b, las publicaciones de profesores y profesoras se reparten de forma relativamente parecida en las cinco macrocategorías contempladas por Scopus (Multidisciplinary, Health Sciences, Life Sciences, Physical Sciences, Social Sciences) y en la categoría de ByD. Se aprecia una mayor inclinación de las mujeres por el ámbito tecnológico que se encuentra recogido en Physical Sciences (34 \% de las publicaciones frente a $29 \%$ de los hombres), incluso por encima de ámbitos de conocimiento más cercanos como las Social Sciences $(23 \%$ frente a $27 \%$ de los hombres), algo que puede explicarse por una tendencia de las mujeres a trabajar en ámbitos más nuevos del conocimiento (Rhoten y Pfirman, 2007) y que también puede apuntar a una vocación más aplicada de éstas. Por lo demás, las diferencias en la actividad de publicación entre profesores y profesoras, a este nivel, resultan mínimas.

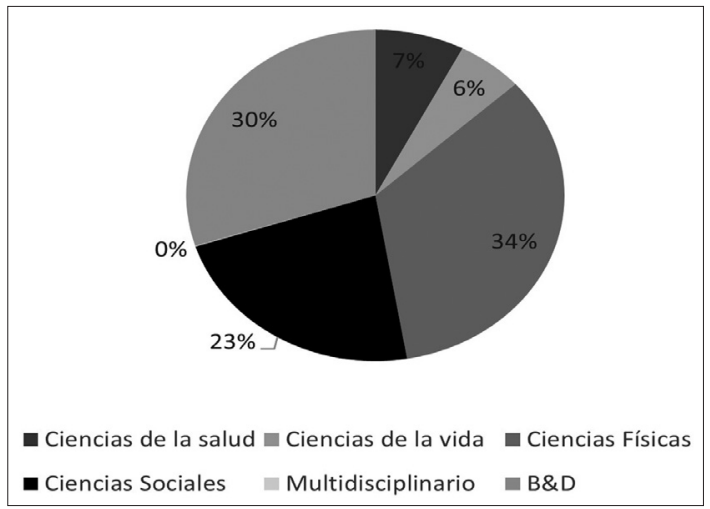

Figura 1a. Publicaciones por grandes áreas - Mujeres 


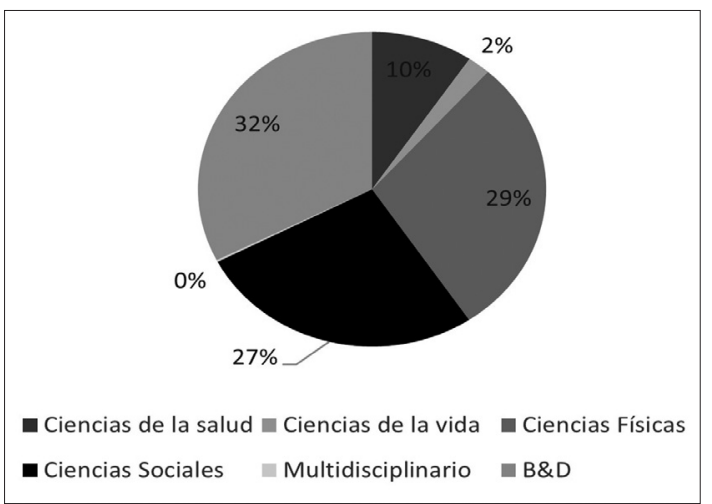

Figura 1b. Publicaciones por grandes áreas - Hombres

La Tabla 2 muestra otros indicadores generales de actividad de los dos conjuntos. Las profesoras publican proporcionalmente menos en acceso abierto y proporcionalmente más bajo licencia de acceso. En promedio, las mujeres incluyen aproximadamente las mismas referencias en sus trabajos que los hombres; sin embargo, necesitan más referencias para obtener una cita respecto a los hombres. Es decir, que para obtener el mismo resultado que los hombres necesitan realizar un mayor trabajo de recopilación de fuentes y de fundamentación de su investigación.

\begin{tabular}{|l|r|r|}
\hline \multicolumn{1}{|c|}{ Producción y actividad } & \multicolumn{1}{c|}{ M } & \multicolumn{1}{c|}{ H } \\
\hline Número total de publicaciones de acceso abierto & 195 & 319 \\
\hline \% de publicaciones en acceso abierto & $29.3 \%$ & $33.2 \%$ \\
\hline Número total de publicaciones bajo licencia de acceso & 471 & 641 \\
\hline \% de publicaciones bajo licencia de acceso & $70.7 \%$ & $66.8 \%$ \\
\hline Número total de referencias bibliográficas & 17285 & 24126 \\
\hline Media de referencias bibliográficas por publicación & 25.95 & 25.13 \\
\hline Media de referencias bibliográficas por citas recibidas & 3.9 & 2.46 \\
\hline
\end{tabular}

Tabla 2. Datos generales de producción y actividad en revistas de Scopus 
La Tabla 3 muestra los tres indicadores de interdisciplinariedad encontrados para los dos conjuntos de autores y autoras. En general, los hombres presentan una ligera ventaja en variedad, equilibrio y disparidad, aunque, como conjunto, presentan mayores diferencias internas y valores atípicos, atendiendo a la desviación estándar y al rango intercuartílico, con lo cual las diferencias entre hombres y mujeres parecen mínimas.

\begin{tabular}{|l|r|r|r|r|r|c|}
\hline \multirow{2}{*}{} & \multicolumn{2}{|c|}{ Variedad } & \multicolumn{2}{c|}{ Equilibrio } & \multicolumn{2}{c|}{ Disparidad } \\
\cline { 2 - 7 } & M & H & M & H & M & H \\
\hline Media & 0.0182 & 0.0239 & 0.200 & 0.249 & 0.721 & 0.725 \\
\hline DE & 0.0173 & 0.0241 & 0.184 & 0.234 & 0.172 & 0.197 \\
\hline Mediana & 0.0120 & 0.0149 & 0.175 & 0.189 & 0.756 & 0.760 \\
\hline Rango int. & 0.0180 & 0.0240 & 0.240 & 0.313 & 0.213 & 0.171 \\
\hline
\end{tabular}

Tabla 3. Medidas de interdisciplinariedad

Las Figuras $2 a, 2 b$ y $2 c$ muestran la distribución de los dos conjuntos en los tres indicadores, poniendo de manifiesto una mayor dispersión en el colectivo masculino respecto al femenino. En cuanto a la variedad, si ambos conjuntos se concentran en valores próximos al cero, delatando la tendencia a publicar en unas pocas categorías, esta inclinación es ligeramente más marcada en el conjunto femenino (Figura 2a).

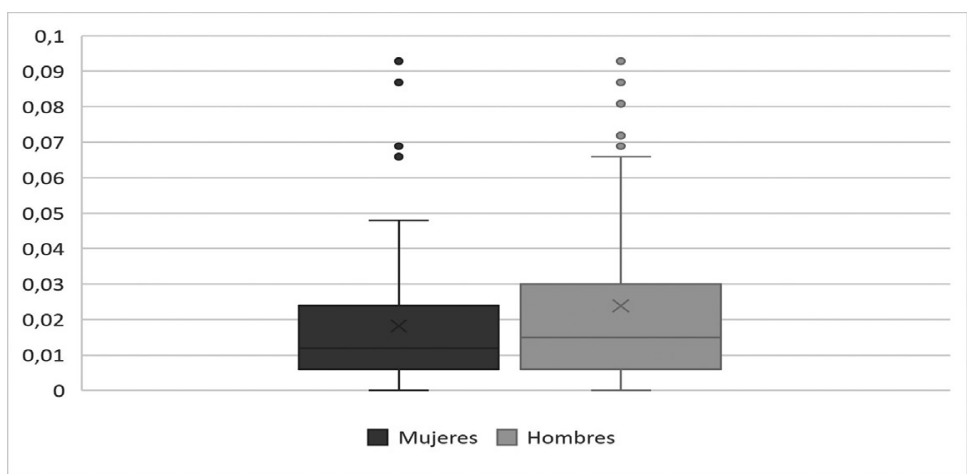

Figura 2a. Distribución de los coeficientes de variedad en los dos conjuntos 
Lo mismo se aprecia en términos de equilibrio, y los dos conjuntos de profesores y profesoras no sólo publican en una proporción limitada de categorías, sino que además tienden a concentrar su producción en algunas, siendo esta tendencia más marcada en las mujeres (Figura 2b).

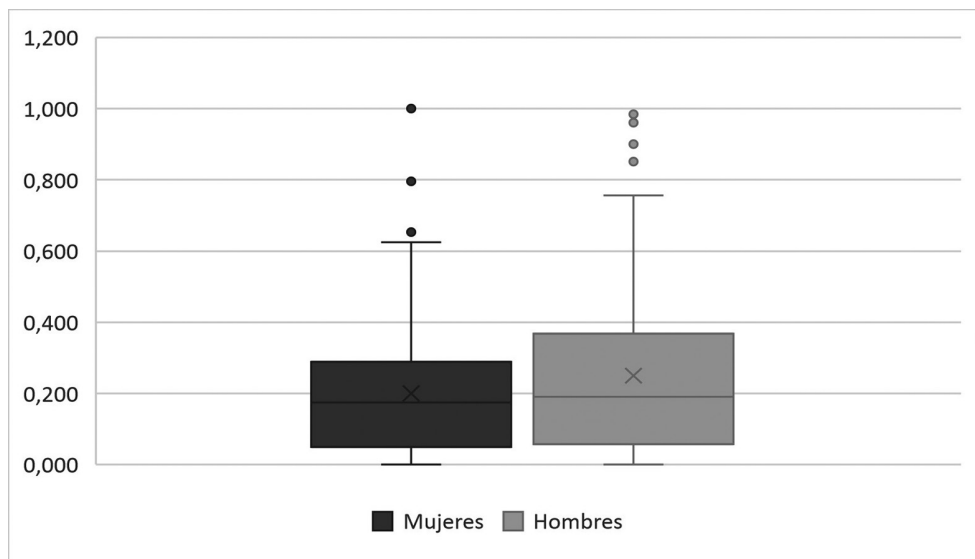

Figura 2b. Distribución de los coeficientes de equilibrio en los dos conjuntos

Finalmente, el análisis de la disparidad revela un conjunto de autoras y autores con líneas temáticas sustancialmente diferenciadas del resto, puesto que en este caso los coeficientes se sitúan más cerca del 1 que del 0 , algo más en el conjunto masculino (Figura 3).

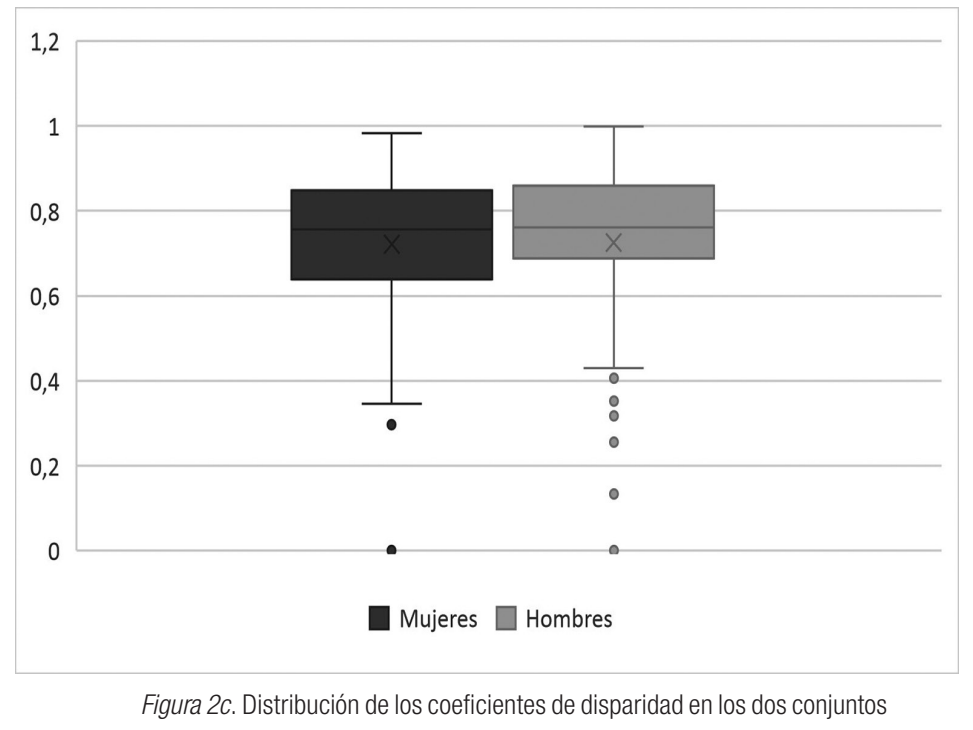

Figura 2c. Distribución de los coeficientes de disparidad en los dos conjuntos 
Para rechazar la hipótesis nula de igualdad entre profesores y profesoras en las tres variables de variedad, equilibrio y disparidad, se realizó la prueba $U$ de Mann Whitney, cuyos resultados se muestran en la Tabla 4.

\begin{tabular}{|l|r|r|}
\hline & U de Mann-Whitney & \multicolumn{2}{|c|}{ Valor de $\boldsymbol{p}$} \\
\hline Disparidad & 5095 & .477 \\
\hline Variedad & 4954 & .251 \\
\hline Equilibrio & 4960 & .258 \\
\hline
\end{tabular}

Tabla 4. Resultados de la prueba U de Mann-Whitney

En ningún caso pudo rechazarse la hipótesis nula de igualdad, situándose los valores de $p$ por encima del 0,05 .

Por otro lado, los coeficientes de variedad y equilibrio en la muestra completa (hombres y mujeres) resultaron correlacionar con el número de documentos (Tabla 5), creciendo a la vez que éstos, explicando en parte la ligera ventaja en esos indicadores de los hombres que en la muestra son más productivos.

\begin{tabular}{|l|r|r|}
\hline & Coeficiente Rho de Spearman & \multicolumn{2}{|c|}{ Valor de $\boldsymbol{p}$} \\
\hline Disparidad & .034 & .628 \\
\hline Variedad & .766 & .000 \\
\hline Equilibrio & .747 & .000 \\
\hline
\end{tabular}

Tabla 5. Resultados de las pruebas de correlación

\section{DiSCUSIÓN Y CONCLUSIONES}

Esta investigación pretendía documentar diferencias en la actividad investigadora de las profesoras frente a los profesores para detectar posibles diferencias cualitativas en la producción cuantitativamente más escasa del colectivo femenino. Con este fin, nos hemos planteado la pregunta de si la producción científica femenina puede considerarse más interdisciplinaria y diversificada temáticamente frente a la masculina, analizando la actividad de publicación del profesorado de ByD con filiación en España y reflejada en Scopus. Las mujeres presentan una ligera desventaja frente a los hombres en términos de presencia en la base de datos, número de publicaciones y proporción de trabajos publicados en abierto, mientras que parecen necesitar un mayor número de referencias para obtener una cita respecto a los hombres. Además, tienden a publicar un poco más que los hombres en revistas del área Physical Sciences. En cuanto a las diferencias relacionadas específicamente 
con la interdisciplinariedad, los resultados muestran que la producción científica femenina no parece más interdisciplinaria que la masculina, por lo menos atendiendo a las categorías de Scopus y al análisis de la diversidad temática de títulos y resúmenes. Los indicadores de variedad y equilibrio tienden a crecer con el número de publicaciones, acentuando el sesgo de productividad a favor de los hombres que tienden a ser más productivos. Por otro lado, la similitud de los indicadores correspondientes a los dos colectivos puede interpretarse también como una característica propia de la disciplina que, en el estudio de Dong et al. (2018), se configura además como un ámbito cada vez más interdisciplinario.

También es importante tener en cuenta las limitaciones del presente estudio, empezando por el propio sistema de clasificación disciplinaria (Scopus), considerando que, según Huang et al. (2021), a veces se necesitan sistemas de clasificación muy granulares para poder apreciar la interdisciplinariedad. Otra limitación que cabe mencionar es la propia manera en la que se ha operacionalizado el concepto de disparidad en el presente estudio. Si por un lado se introdujeron técnicas propias del PNL y la RI para poder atender al contenido temático de la producción de cada autora y autor, en línea con las recomendaciones de la investigación más reciente, por otro lado el análisis se tradujo en la medición estadística de la disparidad de categorías, títulos y resúmenes más que en la valoración de los textos completos. En este sentido, en futuras investigaciones la medición de la disparidad debería buscar fórmulas que reflejen más fielmente las posibles diferencias en el contenido temático de publicaciones de hombres y mujeres, a ser posible trabajando con textos completos. Igualmente, sería recomendable ahondar en las temáticas concretas de investigación tratadas por las mujeres de forma cualitativa y no sólo cuantitativa. El origen de los temas de investigación de las mujeres está a menudo en las experiencias e interacciones de sus vidas de todos los días, chocando con la cultura académica más tradicional centrada en la literatura y convenciones disciplinarias (Gonzales, 2018), y es de esperar que las mujeres presenten perfiles investigadores temáticamente diferentes a los de los hombres.

Finalmente, otras razones que pueden haber impedido una diferenciación clara del trabajo de investigación femenino en el presente estudio incluyen las diferencias en el número de publicaciones analizadas para hombres y mujeres, los sesgos del propio sistema de clasificación utilizado, y el hecho de que hemos analizado una dimensión de la actividad investigadora, la publicación en revistas indexadas, que, por naturaleza, puede ser más conservadora y conforme con las normas de un sistema de comunicación científica que, en cierta literatura de corte feminista, se consideran como masculinas y basadas en valores, como la competición y la búsqueda del estatus, más favorecedores para 
los hombres (Gunter, 2009). En este sentido, se puede añadir que reducir la actividad científica a la publicación en revistas recopiladas en bases de datos como Scopus y WoS puede resultar perjudicial para las mujeres que cada vez más van asumiendo tareas de gestión y docencia a expensas de la investigación o que se implican a menudo en el trabajo académico comprometido con la comunidad (Gonzales, 2018). Para el futuro, es importante demostrar el impacto de actividades académicas y de investigación alternativas a la publicación formal en revistas indexadas, especialmente de aquellas que permitan poner en valor la participación femenina en los procesos de generación y transmisión del conocimiento.

\section{REFERENCIAS}

Abramo, Giovanni, Ciriaco D’Angelo y Flavia Di Costa. 2018. "The effects of gender, age and academic rank on research diversification”. Scientometrics 114 (2): 373-387.

Abramo, Giovanni, Ciriaco D’Angelo y Flavia Di Costa. 2019. "Authorship analysis of specialized vs diversified research output". Journal of Informetrics 13 (2): 564-573.

Abramo, Giovanni, Ciriaco D’Angelo y Gianluca Murgia. 2013. "Gender differences in research collaboration". Journal of Informetrics 7 (4): 811-822.

Abramo, Giovanni, Ciriaco D’Angelo y Lin Zhang. 2018. "A comparison of two approaches for measuring interdisciplinary research output: The disciplinary diversity of authors vs the disciplinary diversity of the reference list". Journal of Informetrics 12 (4): 1182-1193.

Adams, Jonathan, Tamar Loach y Martin Szomszor. 2016. "Interdisciplinary research: Methodologies for identification and assessment. Digital research reports". Digital Science, London.

Araújo, Eduardo B., Nuno A. M. Araújo, André A. Moreira, Hans J. Herrmann y Jose S. Andrade. 2017. "Gender differences in scientific collaborations: Women are more egalitarian than men”. PloS one 12 (5): e0176791.

Barthel, Roland y Roman Seidl. 2017. "Interdisciplinary collaboration between natural and social sciences-Status and trends exemplified in groundwater research". Plos one 12 (1): e0170754.

Beaudry, Catherine y Vincent Larivière. 2016. "Which gender gap? Factors affecting researchers' scientific impact in science and medicine”. Research Policy 45 (9): 1790-1817.

Bromham, Lindell, Russell Dinnage y Xia Hua. 2016. "Interdisciplinary research has consistently lower funding success”. Nature 534 (7609): 684.

Chan, Ho Fai y Benno Torgler. 2020. "Gender differences in performance of top cited scientists by field and country". Scientometrics 125 (3): 2421-2447.

Dong, Kun, Haiyun Xu, Rui Luo, Ling Wei y Shu Fang. 2018. "An integrated method for interdisciplinary topic identification and prediction: a case study on information science and library science". Scientometrics 115 (2): 849-868. 
European Commission. Directorate-General for Research and Innovation. 2018. She Figures 2018. Fecha de consulta: 27 de febrero de 2020. https:/op.europa.eu/en/publication-detail/-/publication/9540ffa1-4478-11e9-a8ed-01aa75ed71a1/language-en

Ghiasi, Gita, Matthew Harsh y Andrea Schiffauerova. 2018. "Inequality and collaboration patterns in Canadian nanotechnology: implications for pro-poor and gender-inclusive policy". Scientometrics 115 (2): 785-815.

Gomes, Janaína y Homero Dewes. 2017. “Disciplinary dimensions and social relevance in the scientific communications on biofuels". Scientometrics 110 (3): 1173-1189.

Gonzales, Leslie D. 2018. "Subverting and minding boundaries: The intellectual work of women". The Journal of Higher Education 89 (5): 677-701.

Gunter, Ramona. 2009. "The Emergence of Gendered Participation Styles in Science-Related Discussions: Implications for Women's Place in Science”. Journal of Women and Minorities in Science and Engineering 15 (1): 53-75.

Hernández-Martín, Estela, Fernando Calle, Juan C. Dueñas, Miguel Holgado y Asunción Gómez-Pérez. 2019. "Participation of women in doctorate, research, innovation, and management activities at Universidad Politécnica de Madrid: analysis of the decade 2006-2016". Scientometrics 120 (3): 1059-1089.

Huang, Ying, Wolfgang Glänzel, Bart Thijs, Alan L. Porter y Lin Zhang. 2021. “The comparison of various similarity measurement approaches on interdisciplinary indicators". FEB Research Report MSI_2102, 1-24.

Jacobs, Jerry A. y Scott Frickel. 2009. "Interdisciplinarity: A critical assessment". Annual review of Sociology 35, 43-65.

Jamali, Hamid R., Alireza Abbasi y Lutz Bornmann. 2020. "Research diversification and its relationship with publication counts and impact: A case study based on Australian professors". Journal of Information Science 46 (1): 131-144.

Knobloch-Westerwick, Silvia y Carroll J. Glynn. 2013. "The Matilda effect-Role congruity effects on scholarly communication: A citation analysis of Communication Research and Journal of Communication articles". Communication Research 40 (1): 3-26.

Larivière, Vicent, Chaoqn Ni, Yves Gingras, Blaise Cronin y Cassidy Sugimoto. 2013. "Bibliometrics: Global gender disparities in science". Nature News 504 (7479): 211.

Larivière, Vicent, Etienne Vignola-Gagné, Christian Villeneuve, Pascal Gélinas y Yeves Gingras. 2011. "Sex differences in research funding, productivity and impact: an analysis of Québec university professors". Scientometrics 87 (3): 483-498.

Leahey, Erin. 2006. "Gender differences in productivity: Research specialization as a missing link”. Gender E Society 20 (6): 754-780.

Leahey, Erin, Christine M. Beckman y Taryn L. Stanko. 2017. "Prominent but less productive: The impact of interdisciplinarity on scientists' research”. Administrative Science Quarterly 62 (1): 105-139.

Leydesdorff, Loet e Ismael Rafols. 2011. "Indicators of the interdisciplinarity of journals: Diversity, centrality, and citations". Journal of Informetrics 5 (1): 87-100.

Meng, Yu. 2018. "Gender distinctions in patenting: Does nanotechnology make a difference?” Scientometrics 114 (3): 971-992.

Meng, Yu y Philip Shapira. 2010. "Women and patenting in nanotechnology: Scale, scope and equity", en Nanotechnology and the challenges of equity, equality and development, editado por Susan E. Cozzens y Jameson Wetmore, 23-46. Dordrecht: Springer. 
Montesi, Michela, Isabel Rodríguez Villaseñor y Fernando Bittencourt Dos Santos. 2019. "Presencia, actividad, visibilidad e interdisciplinariedad del profesorado universitario de Documentación en los medios sociales: una perspectiva de género”. Revista Española de Documentación Cientifica 42 (4): 246.

Nielsen, Mathias Wullum. 2017. "Gender consequences of a national performance-based funding model: new pieces in an old puzzle". Studies in Higher Education 42 (6): 1033-1055.

Porter, Alan e Ismael Rafols. 2009. "Is science becoming more interdisciplinary? Measuring and mapping six research fields over time”. Scientometrics 81 (3): 719-745.

Rhoten, Diana y Stephanie Pfirman. 2007. "Women in interdisciplinary science: Exploring preferences and consequences”. Research policy 36 (1): 56-75.

Solomon, Greg E. A., Stephen Carley y Alan L. Porter. 2016. "How multidisciplinary are the multidisciplinary journals Science and Nature?” PloS one 11 (4): e0152637.

Stjernholm Madsen, B. 2016. Statistics for non-statisticians. 2a. ed. Berlín: Springer.

Sugimoto, Cassidy R., Sam Work, Vincent Larivière y Stefanie Haustein. 2017. "Scholarly use of social media and altmetrics: A review of the literature". Journal of the Association for Information Science and Technology 68 (9): 2037-2062.

Thelwall, Mike y Tamara Nevill. 2019. "No evidence of citation bias as a determinant of STEM gender disparities in US biochemistry, genetics and molecular biology research”. Scientometrics 121 (3): 1793-1801.

Van Rijnsoever, Frank J. y Laurens K. Hessels. 2011. "Factors associated with disciplinary and interdisciplinary research collaboration". Research policy 40 (3): 463-472.

Wang, Jian, Bart Thijs y Wolfgang Glänzel. 2015. "Interdisciplinarity and impact: Distinct effects of variety, balance, and disparity”. PloS one 10 (5): e0127298.

Whittington, Kjersten Bunker. 2018. "A tie is a tie? Gender and network positioning in life science inventor collaboration”. Research Policy 47 (2): 511-526.

Woolley, Richard, Mabel Sánchez-Barrioluengo, Tim Turpin y Jane Marceau. 2014. "Research collaboration in the social sciences: What factors are associated with disciplinary and interdisciplinary collaboration?" Science and Public Policy 42 (4): 567-582.

Zhang, Lin, Ronald Rousseau y Wolfgang Glänzel. 2016. "Diversity of references as an indicator of the interdisciplinarity of journals: Taking similarity between subject fields into account". Journal of the Association for Information Science and Technology 67 (5): 1257-1265.

Zhang, Lin, Beibei Sun, Zaida Chinchilla-Rodríguez, Lixin Chen y Ying Huang. 2018. "Interdisciplinarity and collaboration: on the relationship between disciplinary diversity in departmental affiliations and reference lists". Scientometrics 117 (1): 271-291.

\section{Para citar este texto:}

Blázquez Ochando, Manuel, Michela Montesi e Isabel Villaseñor Rodríguez. 2021. “¿Son las investigadoras de Documentación más interdisciplinarias frente a sus colegas hombres? Un análisis aplicado al profesorado español". Investigación Bibliotecológica: archivonomí, bibliotecología e información 35 (89): 133-149.

http://dx.doi.org/10.22201/iibi.24488321xe.2021.89.58433 